\title{
Semantic Representation and Ease of Predication
}

\author{
Paul de Mornay Davies \\ Centre for Speech and Language, Department of Experimental Psychology, University of \\ Cambridge, Cambridge CB2 3EB, United Kingdom \\ and \\ Elaine Funnell \\ Department of Psychology, Royal Holloway, University of London, Egham, Surrey \\ TW20 OEX, United Kingdom \\ Jones' (1985) Ease of Predication hypothesis, which states that underlying differ- \\ ences in the semantic representation of concrete and abstract words can be explained \\ in terms of disproportionate numbers of semantic predicates, is explored in two \\ experiments. The results suggest that (1) the advantage shown by concrete words \\ in terms of greater number of predicates is only apparent for words of low frequency, \\ and (2) Jones' ease of predication variable does not accurately reflect predicate \\ distributions, or differences in imageability. Rather, it appears to represent differ- \\ ences in concreteness. As such, the validity of this concept as the basis of theories \\ of semantic representation is questioned. Models based on the assumption of a \\ "richer' semantic representation for concrete words are therefore not supported. \\ (C) 2000 Academic Press
}

\section{INTRODUCTION}

There is a prevailing assumption in semantic memory research that the underlying representations of concrete and abstract words differ in terms of "richness," i.e., that the concepts of concrete words consist of more semantic features than those of abstract words. The empirical evidence for this assumption is modest, to say the least, yet it has been the basis of numerous approaches which have attempted to explain concreteness effects and their exaggeration in, for example, the reading performance observed in patients with deep dyslexia (e.g., Plaut \& Shallice, 1993; Barry, 1984; Saffran, 1980; Moss \& Tyler, 1995). The main source of evidence for this notion of differen-

Address correspondence to Paul de Mornay Davies, Psychology Academic Group, School of Social Science, Middlesex University, Enfield EN3 4SF, UK. E-mail: paul32@mdx.ac.uk. 
tial semantic richness is a paper by Jones (1985) in which he claims that concreteness or imageability is a function of a variable which he terms "ease of predication." This is the ease with which any particular word "summons the element representing [it] in semantic memory [which] is associated with a number of features or, more fully, predicates" $(1985$, p.2). He argues that highly imageable words are read and comprehended more readily because they have a higher ease of predication rating: they are assumed to be associated with many more semantic features/predicates. Hence the semantic representation of a concrete term is said to be "richer" than that of an abstract term. It could be argued that more predicates would lead to a greater possibility of errors, since one would have to "select" the correct word from many more possibilities. But Jones' hypothesis is that it is the underlying semantic representation that is composed of more predicates (or features). It is conceivable that such a problem could arise if more than one lexical word form shared the same underlying predicates. Indeed, if two words share all except one defining predicate (e.g., two birds from the same species), the greater the possibility that they may be confused. As the number of predicates which makes up a concept increases, the chances of this occurring decrease. The more predicates that make up a particular concept, therefore, the greater the likelihood that it will have a distinctive representation.

Jones' hypothesis was an attempt to explain the exaggerated concreteness effect observed in the reading performance of many deep dyslexic patients. According to this hypothesis, semantic errors in deep dyslexia (e.g., reading nephew as cousin; Coltheart, Patterson, \& Marshall, 1987) occur when the predicates activated by the lexical word form do not sufficiently specify a concept but simply "narrow down" the semantic field. In terms of Morton's (1969) logogen model, Jones suggests that if the visual input logogen of the input word does not activate the correct set of predicates, an adjacent logogen may be activated, resulting in a visual error (e.g., reading campaign as camping; Coltheart et al., 1987). Words with a low ease of predication rating (low imageability words) are associated with fewer semantic features and are therefore more prone to error when the semantic structure is disrupted. The reading errors associated with abstract words are more likely to be visual than semantic because they have fewer semantic "neighbors" (predicates).

In deep dyslexia, Jones argues, reading occurs via the semantic route on the basis of predicational information. The output of the predicational route is often not identical with the original stimulus word since the meaning of a word is not a precise entity but a diffuse "set" of possibilities (Anderson \& Ortony, 1975). This unstable reading route is normally suppressed in adult readers, and it is only when routes which activate phonology directly from print are damaged, as in deep dyslexia, that it is utilized. Hence deep dyslexic patients make semantic and visual errors in reading and show a strong imageability effect, which Jones argues is really an "ease of predication effect.' He also explains the reverse of this effect in Warrington's (1981) patient 
CAV by suggesting that "the output of the predicational route . . was not suppressed as it normally is, but instead interfered with normal reading processes. Thus the reading of high-imageability words suffered relatively highly from activity along the unreliable predicational route, which interfered with reliable reading processes . . . that had remained intact', (1985, p.16). The resulting conflict between predicational and normal output leads to the excitation of an input logogen adjacent to the original one, producing frequent visual errors, a pattern that was indeed found in CAV's reading. However, this explanation is only relevant to the reversal of the imageability effect in reading; it cannot explain, for example, the abstract word advantage shown by some patients in other tasks requiring semantic processing, such as definition, word-picture matching, and synonymy judgement (e.g., Patient DM; Breedin, Saffran, \& Coslett, 1994).

As Breedin et al. (1994) point out, Jones' argument that the close relationship between imageability and ease of predication reflects the reliance of the former on the latter is no more than an assumption. The high correlation between these two variables (.88) does not imply causality: it is equally plausible that imageability facilitates predication. Indeed, if one reexamines the data, an even higher correlation (.94) is observed between ease of predication and concreteness. It is equally possible, therefore, that the high correlation which Jones reports between imageability and ease of predication is in fact due to the strong association between imageability and concreteness: concrete terms are of necessity also highly imageable, since they are experienced through the senses. Thus an alternative explanation of the observed correlation between imageability and ease of predication in Jones' experiments is that his subjects were influenced in their judgments about ease of predication by the concreteness and/or imageability of the stimulus words.

Definitions of predication in the psychological literature vary (see below) but all refer to more than simply features. Rather, they extend the notion of a relationship between concepts to include any meaningful association of concepts.

\section{Definitions of Predication}

A logical term, signifying any assertion of a relationship between two terms (or psychologically, concepts). (Drever, 1955)

The association of concepts or ideas. (Evans, 1978)

A predicate can be attributed to, or predicated of something. It can represent knowledge, a belief, or an attitude about or toward something. (Ortony, 1979)

Note that these psychological definitions of predication relate to the notion of predicate thought which is:

An element in ... symbolic thought where generalizations concerning concepts are made on the basis of shared features. (Reber, 1985) 
This is very different from the notion of predication in linguistics, which is a formal linking between the subject of a sentence and a proposition that attributes characteristics to that subject. It is the psychological definition, with its emphasis on features that Jones has adopted; but he makes the unfounded assumption of equating predicates and features as one and the same.

The notion that concepts can be decomposed into a set of underlying semantic features originates largely from the work of Rosch and her colleagues (e.g., Rosch \& Mervis, 1975; Rosch, Mervis, Gray, Johnson, \& BoyesBream, 1976). They argue that natural categories can be represented by prototypes (or schemata) of their most characteristic members. Objects in the natural world possess features that cooccur, for example, feathers and wings and beaks, and the pattern of cooccurrence is reflected in the schemata of objects. Thus, a sparrow is a typical bird while a penguin is not: these typicality judgments depend on the exemplars' distance from the schema or prototype. However, it is important to note that Rosch's work deals only with natural objects and artifacts in definable categories. Also, the characteristics of objects are restricted to clearly recognizable features. It is not clear what this theory has to offer to our understanding of artifact terms which do not fall into "natural" categories (e.g., statue), or those which when classified as members of a category (e.g., office equipment; small manipulable objects) do not share enough features to define a prototype. It is also inappropriate for any analysis of those abstract terms that do not belong to categories at all. Any ad hoc attempt to categorize abstract concepts such as "peace," "system," and "luck," results in a rather artificial classification system which is of little or no value. ${ }^{1}$

Nonetheless, Rosch's “'hierarchical taxonomy" with its emphasis on features forms the basis of many contemporary notions of semantic representation (e.g., Plaut \& Shallice, 1993; Durrant-Peatfield, Tyler, Moss, \& Levy, 1997; Devlin, Gonnerman, Andersen \& Seidenberg, 1998). The basic premise (outlined in Rosch et al., 1976) is that in a taxonomy such as living thing $\rightarrow$ animal $\rightarrow$ dog $\rightarrow$ collie, the underlying concepts become increasingly richer in semantic features as one moves down the branch. General (or "superordinate") terms such as animal, vehicle, or furniture elicit imagery less readily because they are assumed to be poorer in figural features (Paivio, 1966). Terms further down the hierarchy, at the "basic level"' (concepts at the highest level which still share a large number of properties, e.g., dog, bird, tree) are more imageable because they comprise all the features of the superordinate plus some additional features (Rosch et al., 1976). For example, the concept bird comprises all the features of animal plus those specific to birds such as wings, feathers, and beak. Lower still, exemplars of bird

\footnotetext{
${ }^{1}$ A notable exception is "emotion words" which are generally easily classifiable. But even here there are gray areas: do words like puzzled, curious, and bored refer to emotional states? (See Clore, Ortony, \& Foss, 1987.)
} 
such as crane will contain extra features like large, long legs, and water$d w e l l i n g$. Although not explicitly stated, the logical extension of this argument is that abstract words must be more "general" since they are low in imageability. Concrete words are assumed to possess a greater number of semantic features than abstract words; the number of features determines the imageability of the concept. This explanation was first developed to explain the exaggerated concreteness effect often exhibited by patients with deep dyslexia (e.g., Coltheart, Patterson, \& Marshall, 1980). However, it is not clear why the ease with which a word elicits mental imagery should influence its readability. Jones (1985) therefore linked this notion to a variable which he termed ease of predication: that is, the ease with which semantic predicates can be generated for a word, which is highly correlated with imageability. But Jones may not have been justified in linking feature-based theories from cognitive psychology with the notion of predication, which is a theoretical construct originating in linguistics. Linguists might argue that these two concepts are not the same, and that predicates are in fact composed of semantic features. For example, Leech (1974) shows how an assertion like: "My uncle owns this car' can be broken down into two arguments ('my uncle' and 'this car') linked by a predicate (owns). He points out that semantic predicates are very different from the predicates of traditional grammar, since "these logico-semantic units [are unlikely] to have any straightforward correspondence with syntactic units" (1974, p.129). For example, in the sentence, "The woman was in front of the car,' the predicate is expressed by 'was in front of' which is not a single syntactic unit. More importantly, a semantic predicate can be broken down into a set of semantic features. In the sentence "Adam boiled an egg," for example, the predicate 'boiled' might be analyzed into three components: $\rightarrow$ COOK, + IN WATER, and + PAST. Hence the linguistic definition of a predicate is more complex than simply a semantic feature; indeed predicates are composed of sets of features. This outlines the theoretical differences between predicates in the linguistic sense and Jones' use of the term "predicates" to mean semantic features. The important question is: are they empirically different?

Many other arguments from psycholinguistics are also germane to this issue. Jackendoff (1992) argues that many abstract concepts like color or temperature (which are highly imageable) also pose a problem for a featurebased account, since they have a continuous (as opposed to a discrete) range of values. How, for example, can colors be decomposed into features? He goes further and argues that even concrete concepts cannot be decomposed into conceptual primitives by recourse to their features. What feature, for example, would distinguish between the lexical entries for "duck" and "goose" since they are both animate, nonhuman things, both are types of bird, types of water fowl, and so on? To distinguish between them on the basis of their appearance (e.g., \pm long neck) is clearly too simplistic and more importantly, the feature \pm long neck does not represent a conceptual 
primitive. Instead Jackendoff argues that "concepthood is a property of conceptual constituents, not conceptual features" (1992, p.43). In his opinion, each conceptual constituent belongs to one of a small set of ontological categories (or conceptual ' "parts of speech') such as Thing, Event, State, Place, Path, Property or Amount. Thus, a concept may consist of "concrete', constituents (Things) as well as more "'abstract"' components (e.g., Properties). As such, no concept is either completely concrete or completely abstract, but is composed of many constituents that are not bounded by such limitations. Nevertheless, it seems likely that object concepts will consist largely of Things while intangible concepts will contain more constituents classified in other categories such as State and Amount. Jackendoff does not say whether any one of these categories contains constituents that produce a more salient semantic representation than any other. In this respect, although this theory questions the veracity of feature-based models of semantics, it still does not explain why words whose concepts consist largely of Things should have any advantage over those whose concepts are chiefly composed of Events, States, or Places.

Although it is often reported that Jones' data support the notion that more predicates can be generated for concrete than abstract words (e.g., Franklin, Howard, \& Patterson, 1995), in fact his subjects were not asked to generate predicates at all. Instead, they were asked to estimate the number of predicates they could produce, if asked, on a 7-point rating scale. There is no indication that Jones' ease of predication ratings map onto the actual predicates of words. In addition, subjects' estimations of their knowledge about a particular item may not be an accurate reflection of their actual level of knowledge. For example, recent research concerning another semantic variable, concept familiarity, suggests that subjects can overestimate their level of knowledge in such rating tasks (Funnell \& de Mornay Davies, 1996). This raises another important point. The level of familiarity or frequency (i.e., how often a word is encountered in the language) may influence subjects' ratings about ease of predication. No attempt was made in Jones' experiment to control for such variables, because there are no reliable effects of word frequency on the reading performance of deep dyslexic patients. However, two of Warrington's (1975) semantic memory patients showed an interaction of frequency and concreteness: EM's concrete word advantage and AB's concrete word deficit were only apparent for words of low frequency. In addition, CAV (Warrington, 1981) showed a concrete word deficit in reading, again only for low frequency words. DM (Breedin et al., 1994) shows a deficit for low frequency concrete words in tasks of definition, word-picture matching, and synonymy judgment. If Jones' ease of predication variable represents differences in the semantic representation of concrete and abstract words, it must also explain the interaction of concreteness and word frequency.

In Jones' experiments the term predication was unfamiliar to his subjects, 
hence the phrase ease of predication was operationalized as "ease of putting words into simple factual statements." In my view, this is not the same as ease of predication. It may produce a bias in favor of concrete words, since they can probably be put into simple factual statements more easily than abstract words, not necessarily because they have richer semantic representations, but simply because of the nature in which they are acquired. The production of "factual statements" implies concrete features: "hard facts" which are usually tangible, perceivable phenomena. Since abstract concepts are usually acquired in the context of language (as opposed to concrete terms that are acquired through the senses) they are less likely to possess "concrete" features, but may possess associations with concrete concepts, which might also be called predicates. In addition, Jones' subjects were given examples to show that "a wide variety of knowledge was eligible for inclusion." But the examples given contrast the concrete word DOG with the abstract word IDEA. Jones lists 11 "factual statements" about DOG while suggesting that the word IDEA "would probably be judged as very difficult to make simple factual statements about.' So difficult, in fact, that he gives no factual statements about it at all. Therefore, both the operational definition of ease of predication and the examples given would bias subjects to give higher ratings to concrete words. The numbers of concrete and abstract stimuli were also unequal. Of the 125 words used in Jones' ease of predication rating experiment, only $24 \%$ were abstract, while $76 \%$ were concrete. It is conceivable that this disproportionate set may have further contributed toward a concrete word bias in subjects' estimations of ease of predication.

Barry (1984) has outlined a model, based on Jones' work, which is also designed to explain the reading pattern observed in deep dyslexia, in particular the exaggerated concreteness effect. Where this model differs from Jones is in its emphasis on the relative range of predicates summoned by a word, rather than the absolute number of predicates. Barry suggests that a concrete word evokes a very narrow range of semantic predicates. The unit corresponding to the target word is maximally activated, while a small number of synonyms and category coordinates will also be activated, but to a lesser degree. For more abstract words, there will be a larger range of activation, with a larger set of units activated above the hypothetical threshold. For very abstract words, there will be a greater range still, with a larger and more varied set of units (including many associatively related links) activated above threshold level, but to a lesser degree such that none are activated to a sufficient degree to accurately specify an output word. If the range of semantic activation is very large (for very abstract words) with no clearly distinguished units within the range being activated to a degree which specifies a candidate word, no response will be made. Barry predicts therefore, that "(i) there should be more responses given to abstract words (i.e. a greater range of responses), and (ii) that there should be more shared-feature than associative types of responses produced to concrete words"' (1984, p.333). 
Since predication is defined as "the association of concepts or ideas" (Evans, 1978), it seems reasonable to assume that if given an adequate definition of predicates, subjects will produce what Barry refers to as "associative type" responses. The aim of Experiment 1 therefore was to have subjects generate either predicates or features to a set of concrete and abstract words, as well as to a subset of Jones' words, in order to compare the relative merits of Jones' and Barry's predictions. In addition, the experiment also set out to establish:

1. If concrete and abstract words do indeed produce different quantities of predicates and/or features. This will be established by comparing the mean number of predicates and features produced by each subject to each of the concrete and abstract stimulus words.

2. If predicates and features are one and the same, as Jones assumes, or if the semantic representation of concrete and abstract words differs in terms of the relative distribution of feature-based and associative type (predicate) terms, as Barry (1984) suggests. This will be assessed by comparing the correlations between number of predicates and features produced to concrete and abstract words, and by examining the most common predicates and features produced.

3. How the number of features/predicates produced correlates with Jones' ease of predication measure as well as other semantic variables such as imageability and concreteness. The number of predicates and features generated to the subset of Jones' words will be compared with their ease of predication ratings and to ratings of concreteness and imageability for these words drawn from the Oxford Psycholinguistic Database (Quinlan, 1992)

\section{EXPERIMENT 1: PREDICATE AND FEATURE GENERATION}

\section{Method}

\section{Subjects}

In total, 180 undergraduate students (42 male, 138 female) participated in the experiment. Subjects' mean age was 22.9 years $(S D=4.87)$. Half the subjects were given the predicate generation instructions and half the feature generation instructions. Because the task is quite time consuming, each subject was only asked to provide predicates or features for 10 words. Thus 15 subjects generated predicates and 15 generated features for each of the 60 target words. All subjects participated voluntarily.

\section{Materials}

The Experimental Set consisted of 20 concrete and 20 abstract nouns selected from Paivio, Yuille, and Madigan (1968). Abstract words (those with a Concreteness rating less than or equal to 3.50 ) had a mean rating of $2.18(S D=.33)$. Concrete words (rated 4.00 and above) had a mean of $5.83(S D=.71)$. Each concrete word was matched for word frequency (Kucera \& Francis, 1967) with an abstract word. Half of the 20 pairs were high frequency and half low frequency. High frequency words had a mean frequency count of 125.10 ( $S D=$ 
79.88); low frequency words had a mean of $15.95(S D=14.31)$. Twenty words were selected from Jones (1985), half of which were concrete and half abstract. Concrete items in the Jones subset had a rating $\geq 3.50$, abstract items $\leq 3.50$, in line with the Experimental Set. These were arranged into six lists of 10 words such that each contained an equal number of concrete, abstract, and Jones' words. Also, during the assignment of words, an effort was made to ensure that no semantic or lexical relationship existed between any of the words on each list. The words were arranged in two different orders of presentation. One word was printed at the top of each page of a 10-page booklet, with a colored front cover containing the instructions for either predicate or feature generation.

In order to devise suitable task instructions, it seemed appropriate to examine previous attempts to assess predicate generation. There have been very few studies that have examined predicate distribution in this manner. Ortony (1979) asked subjects to generate predicates for the subject terms in similes such as "billboards are like warts" and found that they overlapped at a predicate which is frequently produced for the comparator term (warts) but only infrequently generated for the main term (billboards) which in this case was are ugly. Unfortunately, the task instructions used in this experiment were specifically designed for similes.

Rosch et al. (1976) asked subjects to list "attributes" of concrete terms only, since they were investigating how natural kind terms are organized into basic categories in a hierarchical semantic system. The instructions given to subjects specified featural aspects of (concrete) living things (see Appendix 1). These instructions were used as the basis for both the predicate and feature generation instructions in the current experiment, with some modifications. First, the instructions from Rosch et al. explicitly state that subjects are not to produce word associations. But perhaps these would be more appropriate than attributes to tap a system of predicates that are, after all, "'associations of concepts or ideas." de Groot (1989) used word association tasks to investigate aspects of imageability and frequency because of "the commonly held assumption that associations are relatively pure indicators of the way that knowledge is mentally represented ... and on the conception of word association as the retrieval of information along links in the memory network' (1989, p.824). Unfortunately, an inherent problem of word association responses is that they often contain lexical cooccurrences such as "coffeecup" and compound words like "business-man'" which are related at the lexical but not the semantic level. Indeed, a pilot study indicated that this was the case. The instructions were therefore modified to allow associative responses, and any nonsemantic responses were removed from the corpus during analysis. Instead, subjects were asked to avoid "personal or idiosyncratic associations" (see Appendix 2). The first paragraph of the instructions for feature generation was very similar to the original Rosch et al. instructions, except that the term "attributes" was replaced with "features"' and "objects" was replaced with "concepts." Also, examples were given of possible features for an abstract as well as a concrete concept. For the predicate generation instructions, the first paragraph contained a definition and description of predicates based on the definitions cited earlier, as well as examples of concrete and abstract concept predicates.

\section{Procedure}

Two groups of 90 subjects participated in the experiment. Each group received either predicate generation or feature generation instructions. For each group, the procedure was identical. The booklets containing the abstract, concrete, and Jones' word stimuli were distributed among the subjects at the end of a lecture period, ensuring that no two neighboring subjects received the same booklet. The front cover of each of the six booklets was of a different color so that this would be easily noticeable. The nature and purpose of the experiment was explained, and the instructions read aloud by the experimenter, allowing time for any questions. At a given signal, the subjects began to generate predicates or features to the first word. They were stopped by the experimenter after $90 \mathrm{~s}$ and told to move on to the next word. This procedure was repeated for the remaining items until all 10 words had been presented. 
TABLE 1

Mean Number of Predicates per Subject per

Word: High and Low Frequency

\begin{tabular}{llc}
\hline & \multicolumn{2}{c}{ Number of predicates } \\
\cline { 2 - 3 } & HF & LF \\
\hline Concrete & 5.83 & 6.59 \\
Abstract & 4.74 & 3.90 \\
\hline
\end{tabular}

\section{Results and Discussion}

The predicate and feature responses given to the Experimental Set were collated and arranged in order of frequency. Any response given by only one subject was classified as idiosyncratic and removed from any further calculations. Any response given by two or more subjects was classified as frequent. This measure was taken in order to eliminate any responses that were associated with the stimulus word for personal, or idiosyncratic, reasons by any one subject. Both concrete and abstract words produced an equal proportion of idiosyncratic and frequent responses in an approximately 70 : 30 ratio. The range of frequency ( 2 to 15 responses) was also equivalent across groups.

\section{Ease of Predication and Number of Generated Predicates}

For each stimulus word, the mean number of frequent predicates and features per subject was calculated; thus each word was assigned a PRED (mean number of predicates) and a FEAT (mean number of features) value which essentially represent the "core concept" of that word (cf. Miller \& JohnsonLaird, 1976). Since Jones' hypothesis specifically concerns predicates rather than features, the number of predicates generated will be examined first. Examination of the number of features generated will be deferred until Section 2. The mean PRED scores for the concrete and abstract stimuli were 6.21 and 4.32 , respectively.

The difference between the number of predicates produced to the concrete and abstract words is highly significant (independent $t$ test: $t[38]=4.34$, $p<.001$ ). The frequency of the words also affects predicate generation (see Table 1). Results of a $2 \times 2$ ANOVA with PRED score as the dependent variable revealed a highly significant main effect of concreteness $(F[1,39]$ $=23.61, p<.001)$ but also a significant interaction between concreteness and frequency $(F[1,39]=4.83, p<.05)$. While the difference between abstract and concrete items remains significant in the low frequency group (Analysis of simple main effects: $F[1,19]=27.33, p<.001$ ), in the high frequency group this difference is not significant $(F[1,19]=2.55, p>$ $0.05)$. 
The concrete word advantage in terms of predicate generation only holds when the target words are of low frequency. While in the abstract group there is a tendency for higher frequency items to produce more predicates than lower frequency items, this pattern is reversed in the concrete group; i.e., low frequency items produce more predicates than high frequency items. The pattern of predicate generation is therefore affected by the interaction of concreteness and frequency such that LF Concrete $>\mathrm{HF}$ Concrete $>\mathrm{HF}$ Abstract $>$ LF Abstract where " $>$ " refers to the mean number of generated predicates per subject per word.

\section{Predicates vs Features}

In the analyses reported thus far, generated features and generated predicates follow the same pattern. While, generally speaking, more predicates than features are generated for all words, this difference does not reach significance in any analysis. Although more predicates than features were produced for both the concrete and the abstract words, the difference in both cases is not significant (Concrete: independent $t$ test, $t[38]=0.62, p>.05$; Abstract: independent $t$ test, $t[38]=1.06, p>.05)$. Thus it would appear that subjects are able to generate many more predicates and features to concrete than abstract words, but within the word sets, the number of predicates and features does not differ significantly. These findings have implications for Barry's predictions that will be deferred until the general discussion.

Aside from the absolute PRED and FEAT scores, it is important to be aware of how the number of predicates produced compares with the number of features produced item-by-item within the two groups of words. The number of predicates and features produced to the concrete items is highly correlated $(r=0.87, p<.01)$ while the correlation for abstract words is significantly lower ( $r=.50, p<.05$; Fisher's transform: $Z=2.285, p<.05$ ). This suggests that many more items in the abstract word group produced a differential proportion of predicates and features than in the concrete group. That is to say, if a concrete word summons many predicates, it will also produce many features, but if an abstract word summons many predicates, the number of features that can be produced to it cannot be reliably predicted. Thus, Jones' assumption that predicates and features are one and the same (as measured by number of predicates and features produced) is not supported. This can only be said to be the case for concrete words; for abstract words, predicates are not simply features of that concept. This finding is explored further in the qualitative analysis of primary predicate and feature responses.

For each of the 40 concrete and abstract words in the Experimental Set, the frequency with which predicates and features were produced was examined, in order to establish the "Primary Predicate"' and "Primary Feature" responses. These were the responses produced by the most subjects to each 
TABLE 2

Mean Number of PPF and PP/PF Responses

\begin{tabular}{lrrr}
\hline & PPF & PP/PF & Total \\
\hline Concrete words & 13 & 7 & 20 \\
Abstract words & 9 & 11 & 20 \\
\hline
\end{tabular}

stimulus word. In many cases, the Primary Predicate (PP) and Primary Feature (PF) were the same. For example, in response to the word ELEPHANT both the Primary Predicate and the Primary Feature response was trunk. In such cases, the stimulus item is labeled PPF (Primary Predicate/Feature). For other items, different words were produced as Primary Predicate and Primary Feature responses. The word CHILD, for example, produced the Primary Predicate response toys and the Primary Feature response small. Stimulus items producing different PP and PF responses are labeled PP/PF (Primary Predicate/Primary Feature). Thus the abstract and concrete words can be divided into PPF and PP/PF groups. The number of PPF vs PP/PF items in the two groups is shown in Table 2. As Table 2 shows, the proportion of PPF to PP/PF responses is higher in the concrete than the abstract group. However, this difference is not significant $\left(\chi^{2}[1]=1.61, p>.05\right)$. Thus, although the number of predicates and features produced is more highly correlated for the concrete than the abstract items, this result may not necessarily reflect a higher production of the same responses produced as predicates and as features in the concrete group (at least in terms of the most frequent items produced).

\section{Ease of Predication Ratings and Predicate Distributions}

PRED scores were also calculated for the Jones' subset. A comparison between these figures and Jones' original ease of predication ratings (EoP) revealed a highly significant correlation with the number of predicates generated ( $r=0.85, p<.01$; see Fig. 1a). When correlations are obtained separately for concrete and abstract words, the correlations between EoP and PRED are not significant for either concrete $(r=0.11, p>.05)$ or abstract words $(r=0.29, p>.05$; see Fig. 1b). It appears that the significant correlation found when concrete and abstract words are combined only occurs because these two word classes form two distinct populations which overlap partially in terms of number of predicates, but are separated completely on the EoP scale. Within neither group is the correlation between EoP and PRED score significant.

The following points arise from the results presented thus far.

1. Even when concrete and abstract words overlap in terms of number of generated predicates, there is a huge discrepancy in EoP ratings, favoring concrete words. 


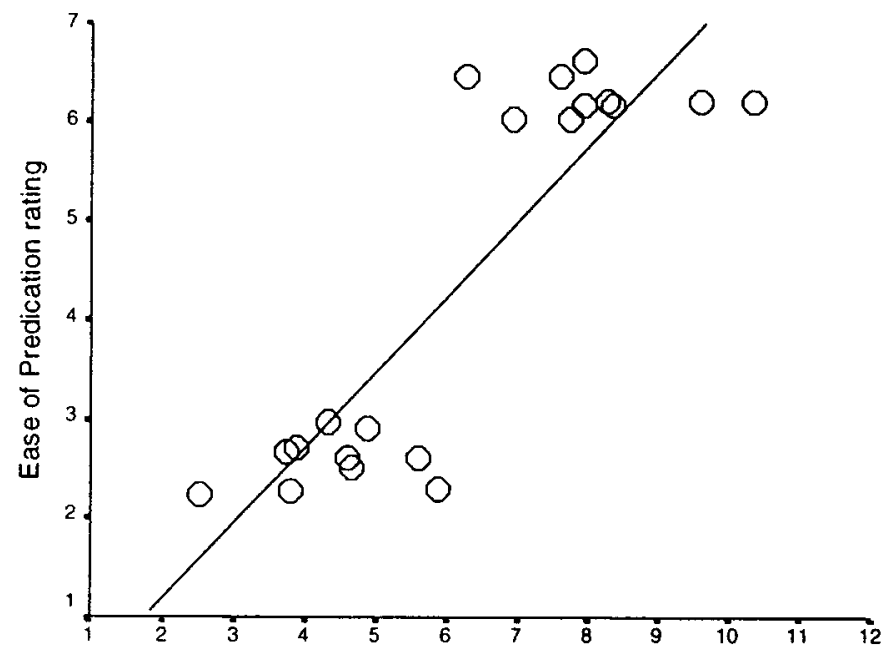

a

Number of Predicates

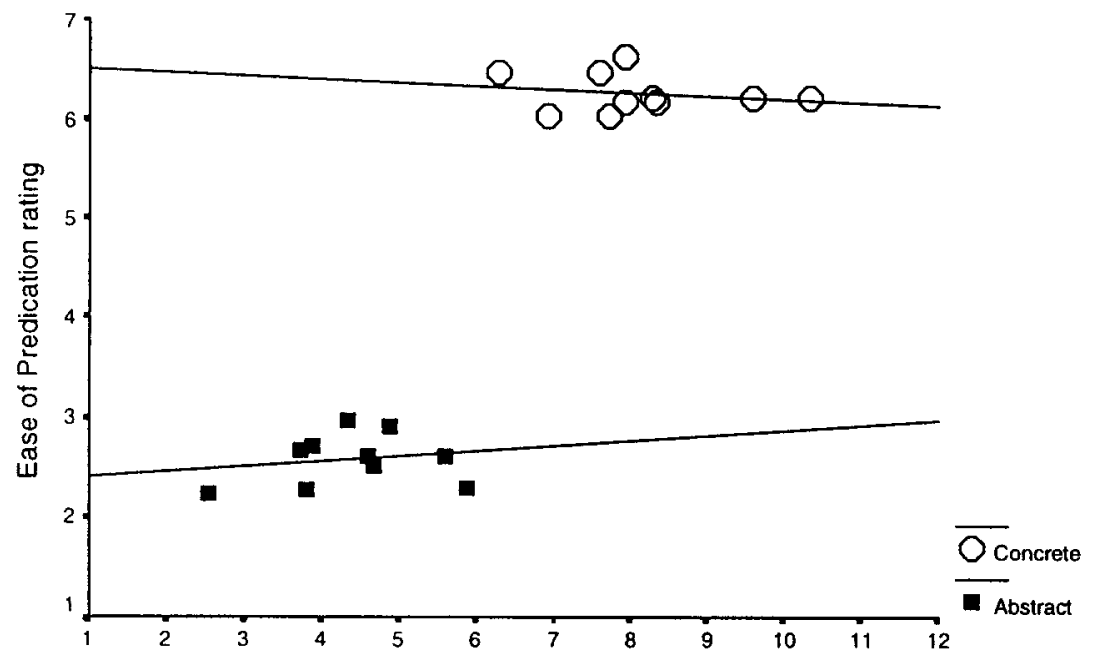

b

\section{Number of Predicates}

FIG. 1. (a) Ease of predication vs number of generated predicates: Jones subset. (b) Ease of predication vs number of generated predicates: Jones subset, concrete and abstract words. 
2. The significant correlation between EoP and PRED score for the complete Jones subset reflects the fact that overall, concrete words are associated with more predicates than abstract words.

3. The marked difference in EoP between concrete and abstract words is not clearly reflected by their respective predicate distributions.

It therefore seems a logical step to examine the relationship between EoP and concreteness. For the complete subset, the overall correlation between EoP and concreteness is higher than that between EoP and PRED $(r=0.99$, $p<.001)$ and the difference between these correlations is highly significant (Fisher's transform: $Z=4.39, p<.001$; see Fig. 2a). The correlations remain significant in both the abstract and the concrete groups (Concrete: $r=0.55$, $p<.05$; Abstract: $r=0.61, p<.05)$ which suggests that rather than reflecting predicate or feature distribution, ease of predication ratings are a better index of concreteness. Jones' ease of predication measure was originally designed as a semantic variable which could explain imageability effects in the reading performance of deep dyslexic patients, although it has since become the basis of many models of semantic representation (e.g., Plaut \& Shallice, 1993). Since ratings of imageability are often highly correlated with those of concreteness, it is perhaps not surprising that EoP correlates with concreteness. However, it is important to examine the direct relationship between EoP and imageability. For the complete subset, the correlation between EoP and imageability is highly significant $(r=0.87, p$ $<.01$ ), but when the concrete and abstract words are examined separately, this correlation disappears. (Concrete: $r=0.02, p>.05$; Abstract: $r=0.07$, $p>.05$; see Fig. 2b). Thus it would appear that, at least for this subset of Jones' words, EoP does not accurately reflect imageability, but rather concreteness. A reanalysis of Jones' original complete set of 125 words in separate concrete and abstract groups lends support to this finding. Concrete words do indeed have a significantly higher mean EoP rating than abstract words $($ Concrete mean $=5.30$, Abstract mean $=2.80$; Mann-Whitney $U=$ $73, p<.00)$. However, while the correlation between EoP and imageability is significant for concrete words $(r=0.85, p<.01)$, it is not significant for abstract words $(r=0.00, p>.05$. Yet the correlation between EoP and concreteness in Jones' original set is significant for both the concrete ( $r=$ $0.89, p<.01)$ and the abstract words $(r=0.59, p<.01)$.

The close relationship between EoP and imageability that Jones talked of is therefore only apparent for concrete words, while that between EoP and concreteness remains strong for both concrete and abstract words. It is therefore likely that Jones' subjects were more influenced in their ratings by the concreteness of a word than by its imageability. There is little evidence to support Jones' assertion that "the ease of predication measure therefore provides ... evidence in favour of the frequently voiced hypothesis that apparent effects of imageability may be mediated via a semantically defined variable [ease of predication] with which it is very closely correlated', $(1985$, p.7). 


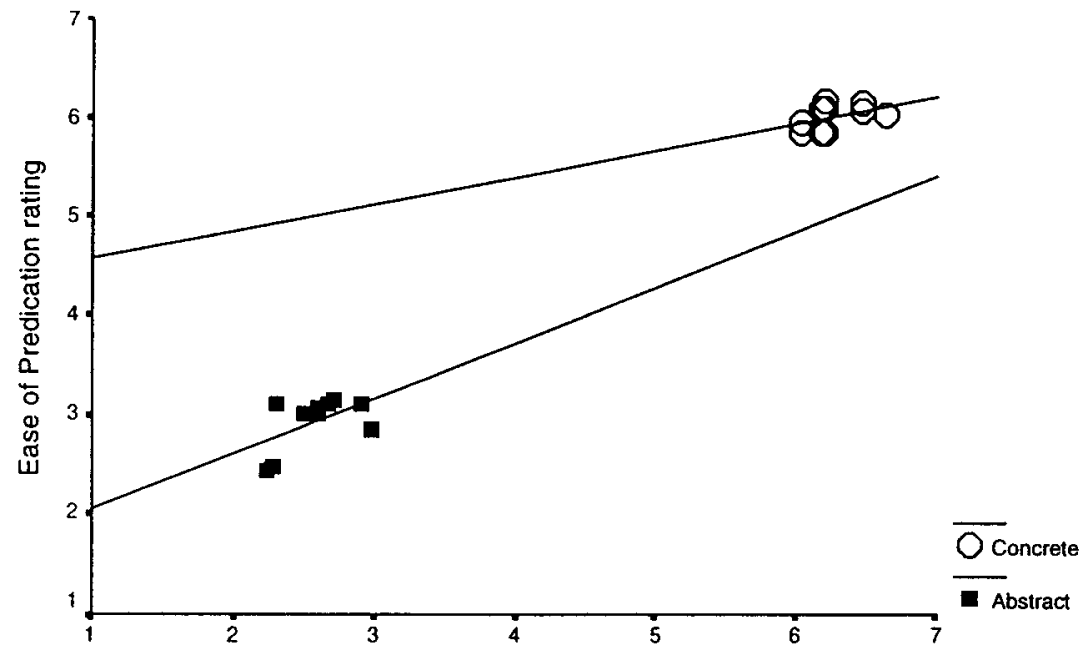

a

Concreteness

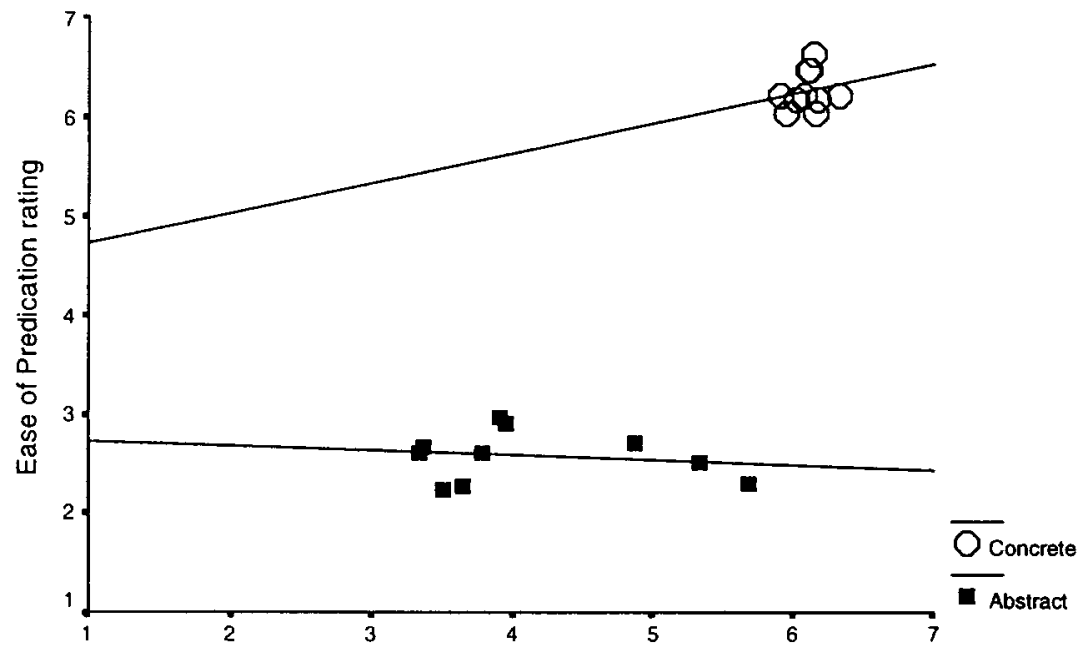

b

Imageability

FIG. 2. (a) Ease of predication vs concreteness: Jones subset. (b) Ease of predication vs imageability: Jones subset. 
TABLE 3

Concreteness Values of Jones Subset vs Concrete and Abstract Experimental Set

Jones subset

Experimental set

\begin{tabular}{llllll}
\hline \multicolumn{5}{c}{ Concrete items } \\
Mean & $S D$ & Range & Mean & SD & Range \\
5.98 & 0.13 & $5.81-6.17$ & 5.83 & 0.71 & $4.06-6.68$ \\
& \multicolumn{7}{c}{ Abstract items } \\
Mean & $S D$ & Range & Mean & SD & Range \\
2.90 & 0.27 & $2.43-3.15$ & 2.82 & 0.33 & $2.26-3.49$ \\
\hline
\end{tabular}

The ease of predication variable essentially represents no more than an alternative measure of concreteness, with one rating substituted for another, and as such it does not provide an adequate explanation of imageability effects in terms of semantic representation.

Although the two variables of imageability and concreteness are closely related, it is important to remember that they are not synonyms. While concrete words are almost always rated as being high in imageability, many abstract words also exist which, while having low concreteness ratings, are also given high imageability ratings. Two such words appeared in the Jones subset. LOVE and JOY both have high imageability ratings (5.69 and 5.33, respectively), but this is not reflected in their EoP ratings (2.30 and 2.50) which are more in line with the concreteness values for these words (3.11 and 3.00). Moreover, in Jones' complete set of 125 words, the correlation between imageability and concreteness is highly significant for the concrete words ( $r=0.83, p<.01)$, but for the abstract words there is no significant correlation $(r=0.13, p>.05)$. It therefore appears that EoP only reflects imageability when imageability is highly correlated with concreteness. This tends to be the case for concrete, but not abstract words.

\section{EXPERIMENT 2: EASE OF PREDICATION RATINGS}

Given that, in the Jones subset, when concrete and abstract words are combined, the high correlation between EoP and number of generated predicates appears to be a spurious effect of the dichotomous concrete and abstract sets, it was hypothesized that if the to-be-rated words consisted of a broader range of concreteness values within each group so that the two distributions were less distinct, this effect would be considerably reduced. In the Jones subset, the range of concreteness values for both concrete and abstract words was considerably less than those of the concrete and abstract Experimental Set, even though the means were very close (see Table 3). Experiment 2 was therefore designed as a replication of Jones' original ease of predication experiment, but with a more evenly distributed range of concrete and abstract words. The purpose of this experiment was to acquire ease of predication ratings for the 40 concrete and abstract experimental words used in Experi- 
ment 1 . In this set of words, the concreteness ratings for concrete and abstract words formed a continuum rather than two distinct sets. In order to ensure that the ratings collected were in line with those of Jones' original ratings, the subset of 20 items from Jones (1985) used in Experiment 1 was also included for evaluation. The aims of this experiment were twofold. First, to provide data for the comparison of the correlations between ease of predication and imageability and concreteness with Jones' data. Secondly, to discover whether the results of Experiment 1 were influenced by the narrow range of concreteness values in the Jones subset.

\section{Method}

\section{Subjects}

Thirty-eight undergraduate psychology students (14 male, 24 female) participated in Experiment 2. Subjects' mean age was 22.7 years $(S D=4.59)$. None of the subjects had participated in Experiment 1.

\section{Materials}

Each subject was presented with the list of 60 words used in Experiment 1 (which included a subset of 20 words from Jones' original experiment) to evaluate for ease of predication. (In line with Jones' experiment, ease of predication was operationalized as "ease of putting into factual statements.') The 60 words were randomized and arranged into two different orders of presentation, with the order for one-half of the subjects being the exact reverse of that for the other half. Alongside each word were printed the numbers 1 to 7 which served as the rating scale. The instructions (from Jones, 1985) were printed on the front page of each booklet (see Appendix 3). These were also read aloud by the experimenter at the start of the experiment.

\section{Results and Discussion}

Ease of predication scores were calculated from the mean ratings across subjects for each stimulus word. The reliability of the means was assessed by comparing two randomly selected sets of subjects' ratings. The split-half reliability estimate was found to be $r=.935, p<.000$, which is comparable to that of Jones' data. The ease of predication scores from this experiment (hereafter referred to as EOP2) for the Jones subset were compared with those from Jones' original experiment. It was noted that while EOP2 was largely comparable with Jones' original ratings for the concrete items, there was a marked discrepancy in the two ratings for the abstract items. Subjects in this experiment rated the abstract words as considerably higher in ease of predication than Jones' original subjects. The discrepancy between Jones' original EoP ratings and the new EOP2 scores was calculated by subtracting the former from the latter. The concrete words in the Jones subset had a mean discrepancy of .11 while for the abstract words this figure was .81. The difference between these two sets of discrepancy scores is highly significant (Mann-Whitney $U=10, p<.0001$ ). This suggests that Jones' subjects may have underestimated their ease of predication ratings for the abstract words, 
possibly because the majority (76\%) of the stimulus words in Jones' original set were concrete words.

In addition, the overall correlation between EoP and EOP2 was 0.97 ( $p<$ $.01)$, but while for the concrete items in the Jones subset this figure remained significant $(r=0.69 p<.05)$, for the abstract items it did not $(r=0.36$, $p>.05)$. The two sets of ratings are therefore not equivalent for abstract words. These data suggest that Jones' ease of predication ratings are not a reliable index of imageability, since they are influenced by the concreteness values of the to-be-rated stimulus words. In the concrete and abstract experimental set, the correlation between EOP2 and the number of generated predicates is highly significant $(r=0.75, p<.01)$. Unlike Jones' words, these correlations remain highly significant when the stimuli are separated into concrete and abstract groups (Concrete: $r=0.63, p<.01$; Abstract: $r=$ $0.68, p<.01$; see Fig. 3a).

Unlike Jones' ratings, the EOP2 ratings appear to provide a more accurate estimation of predicate distributions. However, it is important to bear in mind that the crucial difference between the Jones' items and the concrete and abstract Experimental Set is that the latter consist of a wider range of concreteness values. For this reason, it is necessary to examine the correlations between the new EOP2 ratings and concreteness. As was the case for the Jones subset, the correlation between EOP2 and concreteness for the Experimental Set is very high $(r=0.94, p<0.001)$, and the difference between this correlation and that between EOP2 and number of predicates

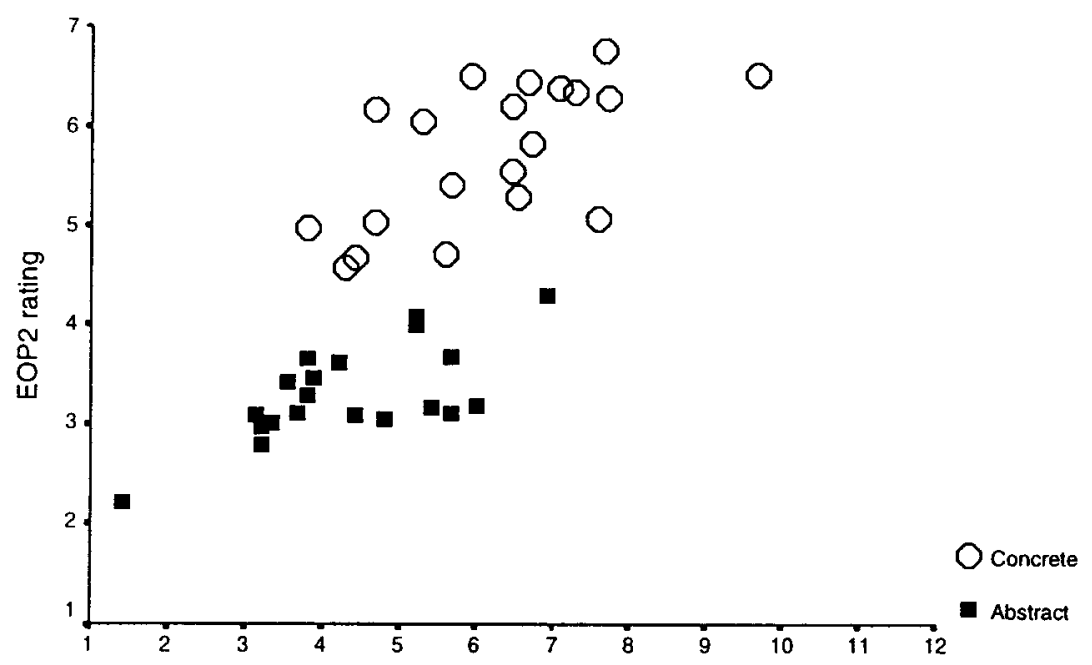

FIG. 3. (a) EOP2 rating vs number of generated predicates: Experimental set. (b) EOP2 rating vs concreteness: Experimental set. (c) EOP2 rating vs imageability: Experimental set. 

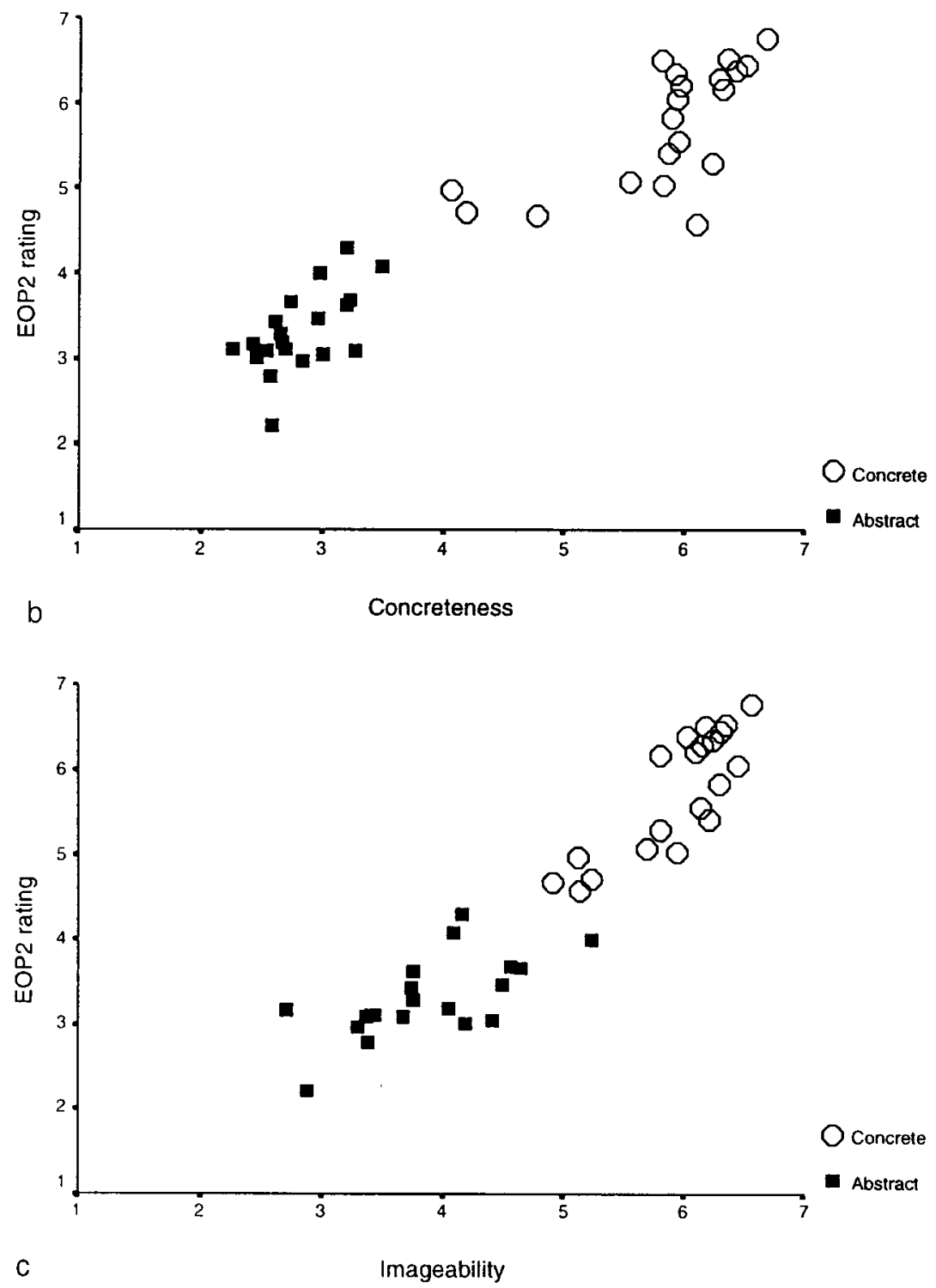

generated is highly significant (Fisher's Transform: $Z=3.29, p<.001$; see Fig. 3b). The correlations between EOP2 and concreteness remain significant when the set is divided into concrete and abstract groups (Concrete: $r$ $=0.68, p<.01$; Abstract: $r=0.62, p<.01)$. As with the Jones subset in Experiment 1 , it is apparent that the ease of predication ratings obtained in this experiment are a reflection of differences in concreteness. The ease of predication ratings in the Experimental Set cover a wider range, in line with 
TABLE 4

Comparison of EoP and EOP2 Correlations with Concreteness and Imageability

\begin{tabular}{lcll}
\hline & Experimental set & \multicolumn{1}{c}{ Jones subset } \\
\hline EOP2 $\times$ concreteness & & & EoP $\times$ concreteness \\
Concrete & $0.68^{* *}$ & 0.41 & $0.55^{*}$ \\
Abstract & $0.62^{* *}$ & $0.52^{*}$ & $0.61^{*}$ \\
EOP2 $\times$ imageability & & & EoP $\times$ imageability \\
Concrete & $0.83^{* *}$ & 0.16 & 0.02 \\
Abstract & $0.65^{* *}$ & $0.74 * *$ & 0.07 \\
\hline
\end{tabular}

$* * p<.01$.

$* p<0.05$.

the broader range of concreteness values in this set, and it is this which makes them a better reflection of predicate distribution than Jones' original ratings.

If we examine the relationship between EOP2 and imageability, it appears that, unlike the Jones subset, the new ease of predication ratings do correlate with imageability. For the complete word set, the correlation between EOP2 and imageability is highly significant $(r=0.94, p<.001)$. The difference between this correlation and that between EOP2 and the number of predicates generated is highly significant (Fisher's Transform: $Z=3.29, p<.001$; see Fig. 3c). When the concrete and abstract words are examined separately, this correlation remains significant in both groups (Concrete: $r=0.83, p<.01$; Abstract: $r=0.65, p<.01$ ). The EOP2 ratings differ from Jones' original ratings in that they do seem to reflect differences in imageability. The discrepancy between EOP2 and EoP for the Jones subset was reported earlier. What is clear from these data is that the discrepancy is significantly more marked in the abstract word set. Also, the high imageability items in this set received a much higher EOP2 rating in this experiment than Jones' original rating. In addition, while there was no significant correlation between the original EoP ratings and imageability for abstract words, $(r=0.07$, $p>.05$ ), for the new EOP2 ratings, this correlation is highly significant $(r=0.74, p<.01)$. This finding is further substantiated by a comparison of EOP2 with concreteness and imageability for the Jones subset (see Table 4). While the correlations between EOP2 and concreteness are broadly similar to those for the Experimental Set, those between EOP2 and imageability are very different. The EOP2 ratings of the abstract words in the Jones subset do appear to reflect imageability, while those of the concrete words do not. It is not clear why EOP2 correlates with imageability in this experiment. It could be that the wider range of concreteness values in the Experimental Set promoted a more accurate estimation of predicate/feature distribution, particularly for abstract words. It is also possible that the significant correlations between EOP2 and imageability in the Experimental Set may have occurred because the broader range of concreteness values increases the pos- 
sibility of imageability and concreteness being correlated. What is clear is that the actual ease of predication rating one estimates is critically dependent upon the distribution, in terms of concreteness, of the stimulus set with which one is presented.

\section{GENERAL DISCUSSION}

The experiments reported here were an attempt to reevaluate Jones' (1985) paper in which he asserted that the apparent effects of imageability in deep dyslexia in fact reflect differences in the underlying semantic representation of concrete and abstract terms, which can be measured in terms of their predicate distributions. This in turn, he claimed, can be estimated using the concept of ease of predication. Experiment 1 required subjects to generate either predicates or features to a set of concrete and abstract words, in order to evaluate the claims made by Jones regarding ease of predication as the basis of concrete-abstract differences in semantic representation. Experiment 2 was a replication of Jones' original ease of predication experiment with a stimulus set which, unlike Jones' original set, contained a wide range of concrete and abstract words. While some of Jones' claims are supported by the results of this study, many of his assumptions have been undermined.

Jones' assumption that concrete words would produce more predicates and/or features than abstract words has received some support from the present data. Subjects generated more predicates and features to concrete than abstract words, but within each group, the number of predicates and features did not differ to a significant level. Some justification is provided, therefore, for Jones' assertion that concrete words are richer in terms of semantic predicates and features, while Barry's (1984) predictions are not supported. Barry suggested that more responses would be given to abstract words (i.e., a broader range of responses) and that these would be largely associative type responses, as opposed to feature-based types. Concrete words, he suggested, would produce fewer responses (a narrower range) and these would be largely feature-based. From the present data we can see that (i) more responses are given to concrete words, and (ii) fewer shared feature than predicate responses (which could be interpreted as associative) are produced to concrete words. The difference between number of features and number of predicates produced is not significant for either concrete or abstract words. Hence Barry's model of deep dyslexia is not supported by these data.

In Experiment 1, the advantage shown by concrete words in terms of predicate and feature generation was only significant for low frequency words. When the stimulus words were of high frequency, the difference between concrete and abstract words in terms of both predicate and feature production was not significant. The interaction of concreteness and frequency has rarely been examined in cases of semantic breakdown. Funnell and Allport (1987) showed that there were no effects of word frequency on the reading perfor- 
mance of deep dyslexic patients who showed an advantage for concrete words. In contrast, when patients show an abstract word advantage in reading (e.g., CAV; Warrington, 1981) this is often only apparent with low frequency words. AB (Warrington, 1975) and DM (Breedin et al., 1994) also show an advantage for abstract words in definition tasks, again only for low frequency words. The results of Experiment 1 suggest that the concrete word advantage in terms of greater numbers of predicates and features exists only for low frequency words: precisely the group of words for which these patients show a deficit. It therefore seems unlikely that the reverse concreteness effect can be explained in terms of disproportionate quantities of predicates and/or features.

Jones' assumption that predicates and features are one and the same is not supported. The number of predicates and features produced correlates very highly on an item-by-item basis for concrete words, but to a significantly lesser degree for abstract words. Results of a qualitative analysis of the most frequently generated predicates and features also suggest that while the primary predicates and features for concrete words are likely to be the same, those for abstract words are not.

Jones' ease of predication variable does not accurately reflect underlying predicate or feature distribution. Even when concrete and abstract words share the same number of predicates and/or features, there is a huge discrepancy in ease of predication ratings, favoring concrete words. It is also apparent that ease of predication does not reflect differences in imageability, except where imageability is correlated with concreteness. In addition, the results of Experiment 2 showed that the ease of predication rating ascribed to a word is critically dependent on the range of concreteness within the concrete and abstract sets in the to-be-rated words. This strongly suggests that the concept is at best an inaccurate and unreliable measure, at worst, that it is little more than an alternative index of concreteness, which provides little insight into the underlying semantic representations of concrete and abstract terms.

Like many theories of semantic representation, Jones' hypothesis uses what might be termed a "subtraction method" approach, in that abstract words are viewed as lacking some element of semantic representation which concrete words possess. An alternative approach might be to relate the differences in concrete and abstract word representation to the different roles that these words play in the language. Because of the nature in which concrete and abstract concepts are acquired, the meaning of a concrete word is relatively fixed, while that of an abstract word is influenced by the linguistic context in which it appears (Saffran, Bogyo, Schwartz, \& Marin, 1980). This observation led Breedin et al. (1994) to suggest that abstract words fall somewhere between open- and closed-class words in terms of the flexibility with which they fit into many different contexts. They go on to suggest that the internal organization of abstract word meanings is highly structured, but like 
those proposed for verbs by Jackendoff (1992) contain many empty argument slots which are filled only when the word is used in conjunction with other concepts. In this respect, concrete word meanings are more openclassed in that it is easier to add some new piece of information to the relatively simple internal organization of a concrete concept than to modify the meaning of an abstract word. This is clearly a theoretical position which demands considerably more research.

Another point that has emerged from these data is the necessity of incorporating some measure of the frequency with which a concept has been encountered when attempting to investigate the concrete/abstract word distinction. This point is often overlooked because of the lack of reliable frequency effects in the reading performance of deep dyslexic patients. Nevertheless, many patients who show disproportionate levels of impairment for concrete or abstract words do so only for words of low frequency (e.g., Warrington, 1975; Breedin et al., 1994). It is plausible that measures of objective word frequency reflect to some degree the frequency with which a concept is encountered and used, i.e., its level of familiarity. Abstract word representations are therefore more prone to disruption after brain damage because they are, on the whole, less familiar to most people. The reverse concreteness effect which has occasionally been observed in patients with progressive deterioration of cortical structures bilaterally (e.g., AB, Warrington, 1975; DM, Breedin et al., 1994) may occur because with extreme degradation, the representations of many concrete concepts may be reduced to a level equivalent to those of abstract concepts. It may be the case that for these patients, many abstract concepts were more familiar to them in the premorbid state because of frequent use. For example, $A B$ was an accountant before the onset of cerebral atrophy, and DM was an academic with a master's degree. Similarly, RG, a fluent aphasic patient described recently by Marshall (1997) who exhibits some of the same deficits as DM, was also an accountant before suffering a bilateral CVA. Hence, for these patients, the representations of some abstract words may become more salient than those of many concrete words because of their frequent use in the patients' premorbid state. For example, words like supplication and inducement are more likely to be familiar to professional persons (e.g., accountants and academics) than other members of society. This only appears to be the case for low frequency concrete and abstract words. Patients who exhibit a deficit for concrete words seem to have retained intact semantic representations for those concrete words that are of high objective word frequency. Assuming that word frequency reflects the experiential frequency of a concept, then it seems that at least in this case, frequency and concreteness interact. Why, then, are there no reliable effects of word frequency on the reading performance of deep dyslexic patients? If, as Shallice (1988) has suggested, frequency effects are indicative of a degraded semantic store, then they should not appear in deep dyslexia, since this can be explained as a deficit of access to intact semantic representa- 
tions (Tyler, Moss, \& Jennings, 1995). Similarly, patient DRB, who has a comprehension deficit for abstract words which is restricted to the auditory modality, shows no effect of word frequency (Franklin, Howard, \& Patterson, 1994). The authors conclude that DRB's difficulty with abstract words is one of semantic access rather than reflecting impaired representations. The reversal of the concreteness effect tends to be observed in the performance of patients who have a progressive deterioration of the cognitive system, which suggests a gradual degradation of semantic representations. In such cases, frequency is a relevant variable, which interacts with concreteness to produce the unusual pattern of a deficit for low frequency concrete words, but not for high frequency concrete words or abstract words of high or low frequency.

The difference between concrete and abstract words may have less to do with absolute number of semantic predicates or features, and more to do with the distribution of features into patterns which distinguish one concept from another in semantic space. Related concepts may share overlapping features, akin to Wittgenstein's (1953) notion of “'overlapping similarities.' In distributed models of semantic representation (e.g., Plaut \& Shallice, 1993; Martin \& Saffran, 1992) concepts are represented as distributions of micro-features in a semantic network. These micro-features may correspond to the set of attributes that make up each concept. As such, each concept is represented as a specific pattern of activation in semantic space. Although it is only recently that semantic representations have been represented in connectionist models, the idea of distributed semantic representation is not new (see, for example, Allport, 1985). The importance of recent models is their attempts to simulate the effects of disruption to the semantic system observed in conditions such as deep dyslexia (Plaut \& Shallice, 1993) and deep dysphasia (Martin \& Saffran, 1992) by including in their model representations of abstract words. However, as mentioned earlier, the assumptions built into such models regarding the differential representations of concrete and abstract words are based on limited data and may not be justified.

\section{CONCLUSIONS}

1. The advantage shown by concrete over abstract words in terms of number of predicates and features (their "richer', representation) is only apparent for low frequency words. When words are of high frequency, there is no significant difference in predicate/feature distribution between abstract and concrete words. Any model of semantic representation of concrete and abstract words based on differential numbers of predicates or features must therefore take into account word frequency.

2. Such models cannot simply equate predicates and features, as Jackendoff (1992) suggested. Even if Jones' assumptions about ease of predication as the basis of semantic representation were correct, predicates cannot be 
feature-based since there is no correlation between the number of predicates and the number of features generated for abstract words.

3. Jones' ease of predication measure, which was intended to represent the distribution of underlying semantic predicates, does not accurately reflect either predicate or feature distributions. It also does not accurately illustrate differences in imageability. Rather, it appears to represent differences in concreteness. As such, the validity of this concept as the basis of theories of semantic representation of concrete and abstract words should be questioned. Models based on the assumption of a "richer" semantic representation for concrete words (e.g., Plaut \& Shallice, 1993) are therefore not built on a solid foundation.

\section{APPENDIX I}

\section{Instructions for Attribute Generation (from Rosch \& Mervis, 1975)}

This is a very simple experiment to find out the characteristics and attributes that people feel are common to and characteristic of different kinds of ordinary everyday objects. For example, for bicycles you might think of things they have in common like two wheels, pedals, handlebars, you ride on them, they don't use fuel, etc. For dogs you might think of things they have in common like having four legs, barking, having fur, etc.

There are six pages following this one. At the top of each is listed the name of one common object. For each page you'll have a minute and a half to write down all of the attributes of that object that you can think of. But try not to just free associate-for example, if bicycles just happen to remind you of your father, don't write down father.

Okay-you'll have a minute and a half for each page. When I say turn to the next page, read the name of the object and write down the attributes or characteristics you think are characteristic of that object as fast as you can until you're told to turn the page again. $(1975$, p. 578$)$

\section{APPENDIX 2}

\section{Instructions for Feature Generation}

This is a very simple experiment to find out the features that people feel are characteristic of different kinds of everyday concepts. Some of these concepts will be abstract, like tact, while others, like bicycle will be more concrete. For example, when thinking about features for bicycles you might think of things they have in common like two wheels, pedals, handlebars, saddles, etc. For an abstract word like tact you might think of things like positive, personal, difficult, and so on.

There are ten pages following this one. At the top of each is the name of one concept word. For each page you'll have a minute and a half to write down all of the features of that concept that you can think of. But try not to just free associate-for example, if bicycles just happen to remind you of your brother, don't write down brother.

Okay-you'll have a minute and a half for each page. When I say turn to the next page, read the name of the concept and write down the features you think are characteristic of that concept as fast as you can until you're told to turn the page again. All words should be treated as nouns. 


\section{Instructions for Predicate Generation}

This is a very simple experiment to find out about the way in which concepts are organized in semantic memory. In psychology, two meaningfully related words are said to be predicates of one another. Predicates can be facts, beliefs or attitudes about or toward something. Your task is to provide predicates for each of ten concepts. Some of these concepts will be abstract, like tact, while others, like bicycle will be more concrete. For example, when thinking about predicates for bicycles you might think of things like wheel, vehicle, ride them, etc. For an abstract word like tact you might think of things like discretion, sensitive, diplomacy, and so on.

There are ten pages following this one. At the top of each is the name of one concept word. For each page you'll have a minute and a half to write down all of the predicates of that concept that you can think of. But try not to just free associate-for example, if bicycles just happen to remind you of your brother, don't write down brother.

Okay-you'll have a minute and a half for each page. When I say turn to the next page, read the name of the concept and write down the predicates you think are associated with that concept as fast as you can until you're told to turn the page again. All words should be treated as nouns.

\section{APPENDIX 3}

\section{Instructions for Ease of Predication Ratings (Jones, 1985)}

Words differ in the ease with which what they refer to can be described by simple factual statements. Some words can be put into statements quite easily and quickly, while for others this can be done only with difficulty or not at all. The purpose of this experiment is to rate a list of 60 words as to the ease or difficulty with which they can be put into simple factual statements.

As an example, the word "dog" would probably be judged as very easy to make simple factual statements about, because it can readily be put into statements such as the following:

A dog is a type of animal.

A dog often lives in a kennel.

A dog barks when angry.

A dog can be pedigree or mongrel.

A dog has four legs.

A dog is called a puppy when young.

A dog wags its tail when pleased.

A dog can be as small as a chihauhau.

A dog can be as large as a St. Bernard.

A dog sometimes chases a cat.

As a contrasting example, the word "idea" would probably be judged as very difficult to make simple factual statements about.

Because words also differ in many other ways (such as how easy they are to mentally image or to categorize), it is important that in making your ratings you attend only to the ease with which each word can be put into simple factual statements.

Your ratings will be made on a 7-point scale, where 1 is the low end of the ease-of-puttinginto-statements scale, and 7 is the high end of the ease-of-putting-into-statements scale. Make your rating by putting a circle around the number from 1 to 7 that best indicates how easy it is to put the word into simple factual statements. The words that are most difficult to put into statements should be given a rating of 1 ; words that are easiest to put into statements should be given a rating of 7 . Words that are intermediate in ease-of-putting-into-statements should of course be rated appropriately between these two extremes, with a rating of 4 representing the average level of easiness. Feel free to use the entire range of ratings from 1 to 7 , 
but don't be concerned about how often you use a particular rating as long as it represents your true judgment.

Work at a reasonable pace, but try to give for each word your best judgment as to the ease with which it can be put into simple factual statements.

If necessary, you can refer back to these instructions when rating the words on the following pages. Do you have any questions?

\section{REFERENCES}

Allport, D. A. (1985). Distributed memory, modular systems and dysphasia. In S. K. Newman, \& R. Epstein, (Eds.), Current perspectives in dysphasia (pp. 32-60). Edinburgh: Churchill Livingstone.

Anderson, R. C. \& Ortony, A. (1975). On putting apples into bottles-A problem of polysemy. Cognitive Psychology, 7, 167-180.

Barry, C. (1984). Consistency and semantic errors in a deep dyslexic patient. In R. N. Malatesh, \& H. J. Whitaker (Eds.), Dyslexia: A global issue (pp. 311-337). The Hague: Martinus Nijhoff.

Breedin, S. D., Saffran, E. M., \& Coslett, H. B. (1994). Reversal of the concreteness effect in a patient with semantic dementia. Cognitive Neuropsychology, 11, 617-660.

Clore, G. L., Ortony, A., \& Foss, M. A. (1987). The psychological foundations of the affective lexicon. Journal of Personality and Social Psychology, 53, 751-766.

Coltheart, M., Patterson, K., \& Marshall, J. C. (1987). Deep dyslexia since 1980. In M. Coltheart, K. Patterson, and J. C. Marshall (Eds.), Deep dyslexia 2nd ed., (pp. 407-451). London: Routledge \& Kegan Paul.

Coltheart, M., Patterson, K. E., \& Marshall, J. C. (Eds.) (1980). Deep dyslexia. London: Routledge \& Kegan Paul.

de Groot, A. M. B. (1989). Representational aspects of word imageability and word frequency as assessed through word association. Journal of Experimental Psychology: Learning, Memory, and Cognition, 15, 824-845.

Devlin, J. T., Gonnerman, L. M., Andersen, E. S., \& Seidenberg, M. S. (1998). Categoryspecific semantic deficits in focal and widespread brain damage: A computational account. Journal of Cognitive Neuroscience, 10, 77-94.

Drever, J. (1955). A dictionary of psychology. Harmondsworth: Penguin.

Durrant-Peatfield, M. R., Tyler, L. K., Moss, H. M., \& Levy, J. P. (1997). Form, function and distinctiveness: A distributed account of category structure. Paper presented at the British Neuropsychological Society, London, April.

Evans, C. (1978). Psychology: A dictionary of the mind, brain and behaviour. London: Arrow Press.

Franklin, S., Howard, D., \& Patterson, K. E. (1994). Abstract word meaning deafness. Cognitive Neuropsychology, 11, 1-34.

Franklin, S., Howard, D., \& Patterson, K. E. (1995). Abstract word anomia. Cognitive Neuropsychology, 12, 549-566.

Funnell, E., \& Allport, A. (1987) Non-linguistic cognition and word meanings: Neuropsychological exploration of common mechanisms. In A. Allport, D. MacKay, E. Sheerer, \& W. Prinz, (Eds.), Language perception and production (pp. 367-400). London: Academic Press.

Funnell, E., \& de Mornay Davies, P. (1996). JBR: A re-assessment of a category-specific disorder for living things. Neurocase, 2, 461-474. 
Jackendoff, R. (1992). Languages of the mind: Essays on mental representation. Cambridge, MA: MIT Press.

Jones, G. V. (1985). Deep dyslexia, imageability and ease of predication. Brain and Language, 24, 1-19.

Kucera, H., \& Francis, W. N. (1967). Computational analysis of present-day American English. Providence, RI: Brown University Press.

Leech, G. (1974). Semantics. Harmondsworth: Penguin.

Marshall, J. (1997). Semantic jargon in aphasia. Paper presented at the Centre for Speech \& Language, March.

Martin, N., \& Saffran, E. (1992). A computational account of deep dysphasia: Evidence from a single case study. Brain and Language, 43, 240-274.

Miller, G. A., \& Johnson-Laird, P. N. (1976). Language and perception. Cambridge, MA: Harvard University Press.

Morton, J. (1969). The interaction of information in word recognition. Psychological Review, 76, $165-178$.

Morton, J., \& Patterson, K. E. (1980). A new attempt at an interpretation, or, an attempt at a new interpretation. In M. Coltheart, K. E. Patterson, and J. C. Marshall, (Eds.), Deep dyslexia (pp. 91-118). London: Routledge and Kegan Paul.

Moss, H. E., \& Tyler, L. K. (1995). Investigating semantic memory impairments: The contribution of semantic priming. Memory, 3, 359-395.

Ortony, A. (1979). Metaphor and thought. Cambridge: Cambridge University Press.

Paivio, A. (1966). Latency of verbal associations and imagery to noun stimuli as a function of abstractness and generality. Canadian Journal of Psychology, 20, 378-387.

Paivio, A., Yuille, J. D., \& Madigan, S. A. (1968). Concreteness, imagery and meaningfulness values for 925 nouns. Journal of Experimental Psychology: Monograph Supplement, 76, $1-25$.

Plaut, D. C., \& Shallice, T. (1993). Deep dyslexia: A case study of connectionist neuropsychology. Cognitive Neuropsychology, 10, 377-500.

Quinlan, P. T. (1992). The Oxford psycholinguistic database. Oxford: Oxford University Press.

Reber, A. S. (1985). The Penguin dictionary of psychology. Harmondsworth: Penguin.

Rosch, E., \& Mervis, C. B. (1975). Cognitive representations of semantic categories. Journal of Experimental Psychology: General, 104, 192-233.

Rosch, E., Mervis, C. B., Gray, W., Johnson, D., \& Boyes-Braem, P. (1976). Basic objects in natural categories. Cognitive Psychology, 8, 382-439.

Saffran, E. M. (1980). Reading in deep dyslexia is not ideographic. Neuropsychologia, 18, 219-223.

Saffran, E. M., Bogyo, L. C., Schwartz, M. F., \& Marin, O. S. M. (1980). Does deep dyslexia reflect right hemisphere reading? In M. Coltheart, K. E. Patterson, and J. C. Marshall, (Eds.), Deep dyslexia, 1st ed. (pp. 381-406). London: Routledge and Kegan Paul.

Shallice, T. (1988). From neuropsychology to mental structure. Cambridge: Cambridge University Press.

Tyler, L. K., Moss, H. E., \& Jennings, F. (1995). Abstract word deficits in aphasia: Evidence from semantic priming. Neuropsychology, 9, 354-363.

Warrington, E. K. (1975). The selective impairment of semantic memory. Quarterly Journal of Experimental Psychology, 27, 635-657.

Warrington, E. K. (1981). Concrete word dyslexia. British Journal of Psychology, 72, 175-196.

Wittgenstein, L. (1953). Philosophical investigations. New York: Macmillan. (Translated by G. E. M. Anscombe). 